\title{
CAUSALIDADE MATERIAL NA FILOSOFIA DA MATEMÁTICA DE ALBERTO MAGNO
}

\author{
Marco Aurélio Oliveira da Silva ${ }^{1}$
}

RESUMO: No século XIII, podem-se observar várias posições divergentes quanto à compreensão da demonstração geométrica. Para Tomás de Aquino, tratarse-ia de uma simples demonstração por causalidade formal - um silogismo no qual a definição do objeto matemático figuraria como termo médio. Roger Bacon, em contrapartida, propunha a experiência como fundamental para entender a prática matemática. Alberto Magno figura em um meio termo, admitindo tanto a demonstração por causalidade formal quanto a demonstração por causalidade material.

PALAVRAS-CHAVE: Causalidade, Alberto Magno, Aristóteles. 
ABSTRACT: In the thirteenth century, several divergent positions can be observed in the understanding of the geometric demonstration. For Thomas Aquinas, it would be a simple demonstration by formal causality - a syllogism in which the definition of the mathematical object would appear as the middle term. Roger Bacon, on the other hand, proposed the experience as fundamental to understand the mathematical practice. Albert Magnus figures in a middle way, admitting both the demonstration by formal causality and the demonstration by material causality.

KEYWORDS: Causality, Albert Magnus, Aristotle. 
O Século XIII é conhecido como o auge do pensamento escolástico, em grande parte devido ao debate suscitado pela redescoberta do Corpus Aristotelicus. Contudo, Aristóteles não era a única autoridade que movia o pensamento da Europa latina de então. Outros cientistas e matemáticos também estavam na lista de gigantes em cujos ombros os anões medievais deveriam subir. Entre eles, podemos contar Ptolomeu e Euclides.

Alberto Magno, por exemplo, tem esta alcunha justamente por estender seus interesses para muito além das discussões filosóficas e teológicas da hora. Sabe-se que ele além de seus comentários a inúmeras obras aristotélicas, neoplatônicas e textos bíblicos, teria fornecido comentários literais a Ptolomeu e a Euclides. No caso deste último, o comentário aos quatro primeiros livros dos Elementos, tratando da geometria plana, foi encontrado em um manuscrito no Convento Dominicano de Viena, o manuscrito $80 / 45^{2}$, objeto de um artigo de Geyer em 1958, que defendia tratar-se do comentário do santo ao geômetra, até então perdido. A pedido do Instituto Alberto Magno, que cuida da edição da obra completa do autor, Tummers produz a primeira edição desta obra e a publica parcilamente em 1984 e completamente em 2014.

Neste sentido, a descoberta do manuscrito $80 / 45$, com a provável autoria de Alberto Magno, permitiu duas indagações a um leitor contemporâneo interessado na 
recepção latina dos Elementos de Euclides: que papel é dado aos diagramas no primeiro comentário literal do ocidente latino aos Elementos? Como estes diagramas particulares podem justificar a pretensão de universalidade das proposições da geometria baseado em um modelo discursivo de prova, como ocorre com a silogística aristotélica?

Além disso, a recepção da tradução de Geraldo de Cremona ao comentário árabe de Al-Naiziri (Anaritius) moveu Alberto a fazer seu próprio comentário da obra. Historicamente, isto ocorre simultaneamente com a recepção do corpus aristotélico, o que obrigou a Alberto a compreender os diagramas dentro de um modelo de demonstração silogístico aristotélico. Como pretendo expor, esta recepção comum não foi livre de consequências para a filosofia da matemática do santo.

Do ponto de vista do seu contexto histórico de desenvolvimento intelectual, Alberto se opunha ao chamado platonismo de Oxford $^{3}$ - referência à ontologia matemática defendida por seus contemporâneos formados por aquela universidade, Robert Grosseteste e Roger Bacon. Para estes, as entidades matemáticas (ponto, linha, plano) 
tinham uma existência independente da consideração intelectual abstrativa, ou seja, existiam na realidade junto com os corpos. Esta tese tem origem em Boécio ${ }^{4}$, a quem erroneamente se atribuía uma tradução dos Elementos ${ }^{5}$.

Ora, o platonismo de Oxford resolveria de modo mais fácil a questão da exatidão e da generalidade, que a demonstração matemática haure do recurso diagramático ou seja, os entes matemáticos estariam lá, nós é que não os perceberíamos --; contudo, esta postura não era aceita pelas investigações de Alberto. Aqui podemos ver uma influência de Aristóteles, pois esta tese albertiana baseia-se nas suas reflexões sobre a diferença de natureza entre os objetos da Física e da Matemática.

No entanto, quanto à questão da atipicidade da comparação entre a generalidade da geometria e a individualidade dos diagramas, pode-se observar a referência constante que Alberto fazia à régua $\mathrm{e}$ ao compasso nas definições matemáticas. E isto já estava presente em outras obras, mesmo ao comentar Aristóteles ${ }^{6}$. Comentando a Metafísica, por exemplo, Alberto tematiza a distinção entre linha e linha sensível - a primeira, objeto da geometria; e a última, objeto da perspectiva, ou seja, da aplicação da geometria à física -, afirmando que a régua e o compasso não construíam linhas e círculos consoantes às definições de Euclides, pois uma linha sensível pode tocar um círculo sensível em mais de um ponto 7 . Ora, um 
diagrama é sempre feito com linhas sensíveis. Então, o que permitiria as conclusões abstratas dos geômetras?

No caso de Alberto, uma compreensão construtivista e anti-platonizante da geometria de Euclides, se dá por uma dupla fonte: a recepção dos Elementos de Euclides e a recepção dos Analíticos Posteriores de Aristóteles, que levará os pensadores da Idade Média a interpretar as conclusões geométricas em conexão com a processo intelectivo de abstração ${ }^{8}$ e com a demonstração por causalidade formal.

Alberto Magno está na confluência de duas tradições: a herança silogística aristotélica, própria da chamada Logica Vetus, oriunda principalmente do trabalho de Boécio; e a influência da filosofia e de matemática árabe, que chega ao ocidente latino na geração do santo. Com a descoberta do manuscrito Viena $80 / 45$, pode-se observar que a recepção de Euclides se deveu principalmente à recepção que o próprio Alberto fizera do comentador árabe Al-Naiziri ${ }^{9}$. Ora, isto colocou Alberto diante de um dilema: na tradição oriunda de Boécio, embora sem admitir um realismo exagerado de tipo platônico, admitia-se que os objetos matemáticos existiam incorporados com os corpos sensíveis. 
Esta ontologia servia de fundamento a uma teoria abstrativa dos objetos matemáticos, os quais seriam obtidos pelo pensamento a partir dos dados sensíveis. Por isso, o matemático poderia raciocinar sobre uma linha que tem comprimento e não tem largura, embora um diagrama não possa satisfazer estritamente esta definição. Em contrapartida, com a recepção do Comentário árabe de Euclides, Alberto adotou uma postura construtivista em relação à geometria euclidiana, interpretando as definições, ou rationes diffinitivae, como regras para a construção de objetos matemáticos, rejeitando a tese da existência in re destas entidades, classificando esta postura como o erro de Platão -na verdade, o erro seria dos intérpretes contemporâneos de Oxford, como Roberto Grosseteste, Rogério Bacon e Roberto Kilwardby ${ }^{10}$.

Conciliar Euclides com Aristóteles mostra-se para Alberto uma tarefa fundamental, já que além dos Elementos, ele comentara a Metafísica, a Física e todo o Organon. Destes, deve-se ressaltar o comentário aos Analíticos Posteriores, no qual Alberto aborda a prova matemática via causalidade formal, recorrendo ao uso de um diagrama ${ }^{11}$ à la Euclides para a demonstração de um teorema, em um contexto no qual pretende exemplificar a posição de Aristóteles. Isto é de particular importância porque a redação deste comentário data aproximadamente de 1252 a 1256, ao passo que a redação do comentário aos 
Elementos é posterior cerca de 10 anos, entre 1262 e $1263^{12}$. Ou seja, trata-se de um exemplo de como o interesse de Alberto por geometria não é uma eventualidade da redação do seu comentário aos Elementos, mas uma presença marcante na sua obra.

A clássica teoria aristotélica da abstração é uma alternativa à assunção platônica de um mundo separado com universais e entidades matemáticas subsistentes. Contudo, a abstração em geral, e a abstração matemática em particular, não tem uma explicação unânime no aristotelismo escolástico medieval.

A abstração é, na verdade, um ato psicológico que depende de uma tese sobre a estrutura categorial da realidade. Ou seja, para que a mente possa abstrair um universal ou uma entidade matemática, esta entidade deve existir de algum modo na realidade. Ora, isto levou Aristóteles a assumir uma teoria onde as propriedades categoriais (acidentes) ineririam em uma substância.

A dificuldade surge quando precisamos compreender a relação entre as propriedades quantitativas (como comprimento, largura e extremidade) com as propriedades qualitativas físicas (cor, temperatura, etc.). Ora, os 
platônicos de Oxford (na verdade, eram também intérpretes de Aristóteles), como Grosseteste e R. Bacon, defendiam que as propriedades quantitativas existiam na realidade independentemente das propriedades qualitativas, embora inerissem em um mesmo substrato. Isto ajudaria a explicar o procedimento abstrativo em Aristóteles. Contra estes autores, como veremos mais adiante, Alberto propõe uma alternativa eminentemente construtivista.

A distinção boeciana entre o objeto da Física e o da Matemática $^{13}$ era lugar comum na Escolástica, e com Alberto não era diferente: a Física trata do que é móvel, ao passo que a Matemática abstrai o movimento. Ora, a Matemática abstrai o movimento e a matéria sensível. Mas a demonstração matemática precisa de ambos, uma vez que os diagramas são traçados com o auxílio imaginativo. Aliás, os diagramas matemáticos são uma etapa importante da demonstração na geometria. Ou seja, as quantidades tais quais definidas na geometria não existem na realidade, mas são uma construção da imaginação. Neste sentido, Alberto afirma:

[...] os matemáticos não precisam de uma magnitude infinita em ato na sua ciência, pois não apreendem a quantidade de acordo com a existência desta, mas de acordo com a imaginação, e procedem de acordo com o poder da imaginação de compor figuras e 
CAUSALIDADE MATERIAL NA FILOSOFIA DA MATEMÁTICA...

ângulos, e não de acordo com uma propriedade da coisa imaginada. ${ }^{14}$

Ou seja, Alberto pensava as definições da geometria como regras para construção de figuras na imaginação. Para evidenciar este ponto, vejamos os seguintes exemplos:

Se alguém imaginar o movimento do ponto segundo o modo reto, que é o único modo simples de movimento, constituirá comprimento sem largura, isto é a linha. Se com efeito mover-se de modo circular constituirá alguma circunferência, que é a linha circular, como se observa no pé móvel do compasso. $^{15}$

Comparando as duas passagens, que comentam respectivamente Aristóteles e Euclides, podemos observar o seguinte:

1) Embora admita a definição boeciana -de origem aristotélica - da Matemática como a ciência que trata sem movimento daquilo que está no movimento, Alberto pensa as entidades matemáticas construtivamente, por exemplo, definindo a linha em função do "movimento" do ponto. $\mathrm{Ou}$ seja, ele não considera que existam entidades matemáticas fora do pensamento, mas que estas são construções mentais na imaginação com o auxílio das definições. Neste sentido, 
Alberto se inclina mais em direção à tradição árabe de recepção de Euclides;

2) ao definir os objetos matemáticos, Alberto respeita o modelo aristotélico de definição de propriedades acidentais (pois é assim que Aristóteles pensa as propriedades matemáticas: como relativas ao acidente da quantidade), referindo-se à propriedade acidental com referência ao nome abstraído. No exemplo, um determinado tipo de linha é definido em função da "retidão" do movimento do ponto de uma extremidade a outra, o que resultará na definição proposta por Euclides: “comprimento sem largura". Este procedimento é o de produção da ratio diffinitiva (razão definitiva, ou simplesmente, definição) em função do tipo de abstração necessária ao matemático. Isto será fundamental para a noção de causalidade formal, que é um tipo de prova silogística que utiliza a definição produzida como termo médio da demonstração matemática.

Por fim, a explicação da matemática como uma ciência que demonstra por causalidade formal tornou-se lugar comum no modo escolástico de compreender a prova em geometria, o que pode ser observado, por exemplo, no mais famoso aluno de Alberto, Tomás de Aquino, como consta em citação infra.

Neste sentido, a intenção por traz desta caracterização é distinguir o conhecimento matemático do 
conhecimento da natureza. Este não é feito pela definição pois sequer temos uma definição perfeita de espécies naturais --, mas a partir de causas exteriores, as chamadas causas eficiente e final. Ou seja, diferentemente da matemática -que produz uma prova dedutiva --, o conhecimento natural se daria a partir do estabelecimento de relações de causa e efeito, o que seria portanto uma consideração móvel, estranha à matemática.

Contudo, podemos observar posições diversas em filósofos contemporâneos a Alberto Magno, a saber, Roger Bacon e Tomás de Aquino. Para o Doutor Angélico, a matemática procederia apenas por causalidade formal a partir da essência, o que significa fazer uma dedução a partir das definições dentro da teoria silogística aristotélica. Neste sentido, Tomás de Aquino afirma

De fato, nas ciências matemática procede-se apenas pelo que pertence à essência da coisa, visto demonstrarem apenas pela causa formal; por isso, não se demonstra nelas algo de uma coisa por outra coisa, mas pela definição própria daquela coisa. Com efeito, embora se dêem algumas demonstrações a respeito do círculo a partir do triângulo ou reciprocamente, isto não se dá senão na medida em que o triângulo está em potência no círculo e reciprocamente. ${ }^{16}$ 
No jargão escolástico, essência é a contrapartida real da definição, que é uma entidade mental-linguística, de modo que uma demonstração a partir da essência significa uma demonstração a partir das definições. Como Tomás não tem trabalhos técnicos de Matemática, diferentemente de Alberto, o que se pode depreender é que ele esteja se referindo ao fato de as definições nas provas matemáticas exercerem um papel totalmente diverso das definições nas "demonstrações" da Ciência Natural. Definições matemáticas são pontos de partida, suposições iniciais ao lado das noções comuns e postulados na estrutura de Euclides. Ou seja, definições são em sentido lato postulados de uma prova, de modo que são noções admitidas para se obter uma determinada conclusão.

Totalmente diverso é o que se observa na Filosofia Natural. Pois, das definições propriamente ditas, pode-se dizer o seguinte: 1) não temos certeza de possuir definições naturais reais que expressam as essências ou se seriam todas nominais, pois elas são obtidas a posteriori em função dos dados empíricos conhecidos. Mesmo na hipótese de haver definições reais que expressem adequadamente essências naturais, elas não serão suposições iniciais da investigação natural. Definições naturais são resultados, pois primeiro há a percepção sensível de um ente dado e, posteriormente, procura-se as definições da espécie à qual pertence este ente. 
CAUSALIDADE MATERIAL NA FILOSOFIA DA MATEMÁTICA...

Portanto, a definição matemática é um ponto de partida para se tirar conclusões, e, por este motivo, a existência do objeto matemático será problemática. Em contrapartida, no caso das definições naturais, a existência não será problemática, pois a definição é produzida a partir de uma percepção sensível inicial, o que será problemático será a aplicabilidade da definição natural produzida. $\mathrm{Ou}$ seja, no caso da definição natural, uma vez confrontado com diversos entes individuais, há que se classificá-los universalmente por comparação, distinguindo o que pertence a uma espécie, ou o que pertence a outra espécie, em função dos predicáveis conhecidos como gênero e diferença específica, pois há a necessidade de se atingir a essência em função de uma definição real. Contudo, deve-se observar que a própria discussão sobre se a definição expressa a essência só faz sentido nas definições naturais, as quais são produzidos após um processo de observação, comparação e abstração. O procedimento matemático é diverso. Pois não faz sentido admitir uma observação prévia, por exemplo, do triângulo, do círculo ou do quadrado para produzir as respectivas definições.

No caso de Roger Bacon, ao tratar da doutrina da Ciência Experimental, ele reconstruira o primeiro teorema de Euclides para dizer que só haverá certeza quando se traçar o diagrama. Neste sentido, ele afirma 
De fato, a mente de quem possui a mais poderosa demonstração a respeito do triângulo equilátero nunca aderirá à conclusão sem experiência, nem se importará, mas a neglicenciará até que se lhe forneça a experiência pela interseção de dois círculos, de uma das seções dos quais sejam traçadas duas linhas até as extremidades da linha dada; então o homem aceita a conclusão com toda tranquilidade ${ }^{17}$

Para Roger, toda a prova racional precisaria de um experimento para auxiliar na demonstração; e o recurso diagramático da prova geométrica, em um sentido lato, ele denomina experimento (experientia). Depreende-se que no caso de Roger Bacon há já uma percepção de que provas matemáticas não são simples deduções analíticas, como parece ser depreendido da afirmação de Tomá de Aquino apresentada acima. Portanto, é interessante observar que há a percepção de um autor medieval, no início da nova recepção no mundo latino das obras de Euclides, de que o diagrama exerce um papel relevante pelo menos no convencimento de uma prova geométrica.

Neste sentido, diferentemente de Tomás, Roger explica a prova matemática da Geometria não como apenas uma demonstração por causalidade formal a partir da essência, uma simples derivação da definição, mas como algo que em uma certa medida depende da construção 
diagramática, embora no texto de Bacon, a palavra diagrama não ocorra como acontece em textos de Alberto. Neste contexto, observa-se de um lado, Tomás de Aquino com uma afirmação fortemente formalista, de outro lado Roger Bacon chamando atenção para o papel da construção diagramática no contexto da prova, percebendo que a prova simplesmente dedutiva não é suficiente; por fim, Alberto Magno parece estar no meio termo, para ressaltar o papel tanto da demonstração por causalidade formal quanto da demonstração por causalidade material, como ele afirma no comentário aos Analíticos Posteriores. Neste sentido, afirma Alberto Magno que

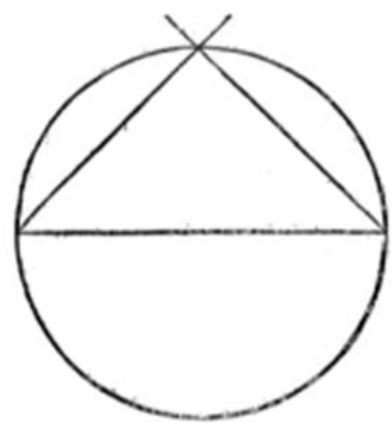

Apresentada a figura, deve-se proceder à demonstração de que o ângulo que está do lado interno do semicírculo é reto; com efeito, o ângulo que está na metade de dois retos é reto; o ângulo 
localizado acima do arco do círculo está na metade de dois retos; portanto, [o ângulo interno] é reto, o que é provado pela causa material ${ }^{18}$.

Causalidade formal é uma demonstração cujo termo médio da prova é a definição do objeto matemático considerado. Em contrapartida, causalidade material é uma prova feita a partir de um outro objeto matemático, como quando se prova algo sobre o triângulo a partir do círculo, ou vice versa. No exemplo de Alberto citado acima, o termo médio é "metade de dois retos", que não consiste na definição de reto e que pressupõe uma determinada relação expressa no diagrama.

Deve-se observar a ligação entre a teoria da prática matemática do Comentário de Alberto Magno aos Analíticos Posteriores, no qual se trata da distinção entre causalidade formal e causalidade material, e o recurso a uma construção diagramática. Isto deve ser assinalado por dois motivos: 1) a distinção entre demonstração por causalidade formal e causalidade material é recorrente, o próprio Tomás se refere à prática matemática como uma demonstração por causalidade formal, enquanto a filosofia natural dependeria de causas extrínsecas, a eficiente e a final; 2) além disso, a transmissão dos Analíticos Posteriores de Aristóteles não tem diagramas; deve-se ressaltar o fato de a transmissão euclidiana depender da construção diagramática, o que é 
replicado por Alberto, além do comentário a Euclides, também em um comentário a um texto aristotélico. Ora, quando Alberto comenta o texto de Aristóteles referido, recorre à construção diagramática típica euclideana, diferente de quando Aristoteles produz provas geométricas. Portanto, partindo da distinção entre causalidade formal e causalidade material, pode-se ressaltar um certo papel emprestado ao diagrama quando se tratar de provas por causalidade material em Geometria.

Contudo, uma dificuldade é que a distinção entre demonstração por causalidade formal e demonstração por causalidade material não parece estar contida explicitamente no comentário de Alberto a Euclides, assunto que pretendo abordar em outra oportunidade ${ }^{19}$.

\section{NOTAS}

${ }^{1}$ Professor Adjunto de Filosofia pela Universidade Federal da Bahia (UFBA). Doutor em Filosofia pela Universidade Federal do Rio de Janeiro (UFRJ).E-mail: silva.marco@ufba.br.

${ }^{2}$ Cf. TUMMERS, 1980, p. 480.

${ }^{3}$ Cf. MOLLAND, 1980, p. 465.

${ }^{4}$ Cf. BOÉCIO, PL 64, 84C-84D. "Atque ut hoc nobis in pervagato exemplo manifestum stt, linea in corpore est aliquid, et id quod est 
corpori debet, hoc est esse suum per corpus retinet, quod docetur ita: si enim separata sit a corpore non subsistit; quis enim unquam sensu ullo separatum a corpore lineam coepit? Sed animus cum confusas res permistasque corporibus in se a sensibus coepit, eas propria vi et cogitatione distinguit".

${ }^{5}$ Cf. TUMMERS, 1980, p. 481.

${ }^{6} \mathrm{Eu}$ apresento um tratamento mais detalhado sobre a relação entre movimento e Geometria na Filosofia de Alberto Magno em SILVA, 2014.

${ }^{7}$ Cf. ALBERTUS MAGNUS, In III Metaph., tr. 2, c. 3. Ed. Col., XVI.1, p. $114 \mathrm{ff}$.

${ }^{8}$ Esta operação produz o que Alberto denomina ratio diffinitiva, ou seja, a definição da entidade matemática.

${ }^{9}$ Cf. BELLO, 2003, p. 229-230.

10 Cf. MOLLAND, 1980, p. 465.

${ }^{11}$ Cf. AlBeRTUS MAGNUS. Liber II Poster. Analyt. tr. 3, c. 2. Ed Borgnet, 2, p. 198b.

${ }^{12}$ Quanto à datação do comentário, sigo Tummers, 1980.

13 BOÉCIO, PL 64, 1250A-1250B. "Nam cum tres sint speculativae partes, naturalis, in motu, inabstracta (...) Mathematica, sine motu, inabstracta (...) Theologica, sine motu, abstracta atque separabilis (...)". ${ }^{14}$ ALBERTUS MAGNUS, Physica, 1. 3, tr.2, c.17; ed. Col, IV, p. 197v4247. Mathematici enim non indigent in sua scientia magnitudine infinita secundum actum, quia non accipiunt quantitatem secundum esse, sed secundum imaginationem, et procedunt secundum potestatem imaginationis componentis figuras et angulos et non secundum potestatem rei imaginatae (...). Cf. MOLLAND, 1980, p. 471.

15 ALBERTUS MAGNUS. Super Euclidem, pr.; ed. Col., XXXIX, p. 1v70-2v2. Si quis enim imaginetur motum puncti quocumque modo secundum rectum - qui solus motus eius simplex est secundum formam -,non constituet nisi longitudinem sine latitudine, et hoc est 
linea. Si vero moveatur circulariter, constituet quidem circumferentiam quae est linea circularis, sicut apparet in pede circini mobili. Cf. BELLO, 2003, p. 4.

16 TOMÁS DE AQUINO. Super De Trinitate, q. 6 a. 1 co. 3; ed. bras., 1999, p. 145. In scientiis enim mathematicis proceditur per ea tantum, quae sunt de essentia rei, cum demonstrent solum per causam formalem; et ideo non demonstratur in eis aliquid de una re per aliam rem, sed per propriam diffinitionem illius rei. Etsi enim aliquae demonstrationes dentur de circulo ex triangulo vel e converso, hoc non est nisi in quantum in circulo est potentia triangulus et e converso.

${ }^{17}$ ROGER BACON. Opus Majus, ed. Bridges, II, p. 168; ed. bras, 2005, p. 189-190. Qui vero habet Demonstrationen potissimam de triangulo equilatero sine experientia nunquam adhaerebit animus conclusioni, nec curabit, sed negliget usquequo detur ei experientia per intersectionem duorum circulorum, a quorum alterutra sectione ducantur duae lineae ad extremitates lineae datae; sed tunc recipit homo conclusionem cum omni quiete.

18 ALBERTUS MAGNUS. Liber II Poster. Analyt. tr. III, c. II; ed. Borgnet, II, p. 198. Sic disposita figura, procedamus sic ad demonstrationem quod angulus in semicirculo stans est rectus: angulus enim qui est medietas duorum rectorum rectus est: angulus super arcum circuli stans est duorum rectorum medietas: ergo est rectus: et probatum est per causam materialem.

${ }^{19} \mathrm{O}$ presente trabalho é resultado de um estágio pós-doutoral realizado no Institut d'Histoire et de Philosophie des Sciences et des Techniques (IHPST/ CNRS - Paris 1 - ENS), no contexto do projeto CAPES/COFECUB “Provas, demonstraç̃oes e representação”. Expresso meus agradecimentos a Marco Panza, Oswaldo Chateaubriand e Abel Lassalle Casanave, que tornaram possível este estágio de pesquisa. Agradeço também à CAPES, que financiou minha estada em Paris. 


\section{REFERÊNCIAS BIBLIOGRÁFICAS}

ALBERTUS MAGNUS. Opera Omnia. Ed. Institutum Alberti Magni Coloniense. Münster i. Westfalen: Aschendorff, 1951.

ALBERTUS MAGNUS. Opera Omnia. Ed. Auguste Borgnet. 38 vols. Paris: Vives, 1890 - 1899.

ALBERTUS, Saint et al. Albertus (Magnus)'commentaar op Euclides' Elementen der geometrie: Expositio Anaritii $X$ primorum librorum geometrie. PMJE Tummers, 1984.

ANDERSON, T. C., Intelligible Matter and the Objects of Mathematics in Aquinas. The New Scholasticism 43, p. 555$576,1969$.

ARISTOTLE Posterior Analitics In BARNES, J. (ed.) The complete works of Aristotle [Translated by Jonathan Barnes]. Oxford: Princeton University Press, p.114-166, 1984.

BACON, Roger. Opera quxedam hactenus inedita. v. 1. Longman, Green, Longman, and Roberts, 1859.

BACON, Roger. The'Opus Majus', of Roger Bacon, Edited by John Henry Bridges,.. Williams and Norgate, 1900.

BACON, Roger. Opus Maius (A ciência experimental). In De Boni, L.A. (org.) Filosofia Medieval. Textos. Tradução de C. A. R. do Nascimento. Porto Alegre: Edipucrs, 2005, p.185-195. 
CAUSALIDADE MATERIAL NA FILOSOFIA DA MATEMÁTICA...

BELLO, Anthony Lo et al. (Ed.). The commentary of AlNayrizi on Book I of Euclid's Elements of geometry: with an introduction on the transmission of Euclid's Elements in the Middle Ages. Brill, 2003.

BELLO, Anthony Lo. Albertus magnus and mathematics: A translation with annotations of those portions of the commentary on Euclid's Elements published by Bernhard Geyer. Historia Mathematica, v. 10, n. 1, p. 3-23, 1983.

BOÉCIO Opera Omnia, in J-P Migne, ed., Patrologia Latina $(\mathrm{PL})$, vol. 64, Paris: Vivès.

BOETHIUS; FOLKERTS, Menso. "Boethius" Geometrie II. Ein mathematisches Lehrbuch des Mittelalters. Von Menso Folkerts. Franz Steiner Verlag. 1970.

CLAGETT, Marshall. The Medieval Latin Translations from the Arabic of the Elements of Euclid, with Special Emphasis on the Versions of Adelard of Bath. Isis, p. 16-42, 1953.

EUCLID. Elementa geometriae. Trans, into Latin by Adelard of Bath, ed. Campanus de Novara. Venice, 1482.

EUCLID. The Thirteen Books of Euclid's Elements. Tr. Thomas Little Heath. Cambridge, 1908.

FOLKERTS, Menso. Euclid in medieval Europe. Benjamin Catalogue for History of Science, 1989.

GEYER, Bernhard. Die mathematischen Schriften des Albertus Magnus. Angelicum, v. 35, p. 159-175, 1958. 
HILBERT, David. The foundations of geometry. Open court publishing Company, 1902.

MANCOSU, Paolo. Philosophy of mathematics and mathematical practice in the seventeenth century. Oxford University Press, 1999.

MAURER, Armand Aquinas on the Foundation of Mathematics. The review of Metaphysics, v. 47, $\mathrm{n}^{\circ} 1, \mathrm{p} .43-61$, 1993.

MOLLAND, A. George. An examination of Bradwardine's geometry. Archive for History of Exact Sciences, v. 19, n. 2, p. 113-175, 1978.

MOLLAND, Andrew G. Mathematics in the thought of Albertus Magnus. In WEISHEIPL, James A. (Ed) Albertus Magnus and the Sciences. Commemorative Essays, p. 463478, 1980.

ROBERT GROSSETESTE, Commentarius in viii libros physicorum Aristotelis. ed. R.C. Dales. Boulder, 1963.

ROBERT GROSSETESTE, In Aristotelis Posteriorum analyticorum libros 1.12 ed.Pamphilus de monte Bononiensis Venice, 1514 (repr. Frankfurt, 1966).

SILVA, M. A. O. Movimento e objetos matemáticos em Alberto Magno. Notae Philosophicae Scientiae Formalis, v. 3, p. 23-31, 2014.

SMITH, V.E. St. Thomas on the object of Geometry. Milwaukee: Marquete University Press, 1954. (The Aquinas Lecture, 1053) 
CAUSALIDADE MATERIAL NA FILOSOFIA DA MATEMÁTICA...

TOMÁS DE AQUINO Sancti Thomae Aquinatis Opera Omnia, Roma-Paris: Leonine edition. 1882-

TOMÁS DE AQUINO. Expositio Super Librum Boethii De Trinitate. Ed. B. Decker. Leiden: E.J. Brill, 1955.

TOMÁS DE AQUINO Comentário ao Tratado da Trindade de Boécio. Questões 5 e 6; Tradução de C. A. R. do Nascimento. São Paulo: Unesp, 1999.

TUMMERS, Paul Marie Joseph Emanuel et al. (Ed.). The Latin translation of Anaritius' Commentary on Euclid's Elements of geometry. Ingenium, 1994.

TUMMERS, Paul MJE. The commentary of Albert on Euclid's Elements of geometry. In WEISHEIPL, James A. (Ed) Albertus Magnus and the sciences: Commemorative essays 1980, p. 479-499, 1980.

WEISHEIPL, J. Athanasius. Albertus Magnus and the Oxford Platonists. In: Proceedings of the American Catholic Philosophical Association. 1958. p. 124-139.

WEISHEIPL, James A. (Ed.). Albertus Magnus and the sciences: commemorative essays 1980. Pims, 198 